\title{
Differentiating pulmonary cavities
}

\section{To the Editor:}

We present the case of a patient with pulmonary cavitation of extremely unusual aetiology, which presented a significant diagnostic challenge despite multidisciplinary involvement. A 54-year-old male presented with a 6-week history of haemoptysis associated with fatigue, night sweats and dyspnoea. His past medical history was significant for coronary artery stenting (treated with aspirin). There was no history of exposure to tuberculosis. He had a 50-pack-year smoking history, prior excessive alcohol consumption and had worked in construction, though denied asbestos exposure. On physical examination, vital signs were normal, his dentition was poor, there was no digital clubbing and chest auscultation was unremarkable.

Initial chest radiography showed a right upper lobe (RUL) cavity. Laboratory testing confirmed a normal full blood count, and renal, bone and coagulation profiles. An anti-neutrophil cytoplasmic antibody (ANCA) test was negative. Levels of $\gamma$-glutamyltransferase $\left(91 \mathrm{IU} \cdot \mathrm{L}^{-1}\right.$, normal range $\left.11-67 \mathrm{IU} \cdot \mathrm{L}^{-1}\right)$, C-reactive protein $\left(215 \mathrm{mg} \cdot \mathrm{L}^{-1}\right.$, normal range $\left.<7 \mathrm{mg} \cdot \mathrm{L}^{-1}\right)$ and lactate dehydrogenase $\left(334 \mathrm{IU} \cdot \mathrm{L}^{-1}\right.$, normal range $120-220 \mathrm{IU} \cdot \mathrm{L}^{-1}$ ) were elevated. Candida albicans was cultured from sputum; blood cultures were sterile and urinalysis was normal. Thoracic computed tomography (CT) demonstrated a $7 \times 5 \times 5$-cm RUL thick-walled cavity abutting the pleura with adjacent nodular lesions (fig. 1). Bronchoscopy was unremarkable and routine culture and testing for mycobacteria from bronchial washings was negative. No evidence of malignancy was demonstrated on bronchial washings, fluoroscopy-guided transbronchial biopsies or percutaneous CT-guided lung biopsy.

Because all specimens were consistently negative for cancer, broad-spectrum antibiotics were initiated for suspected anaerobic infection, with a decision to proceed to surgical lung biopsy if no improvement was observed. However, the patient remained intermittently febrile and subsequently coughed $100 \mathrm{~mL}$ blood during his hospitalisation. Repeat chest CT

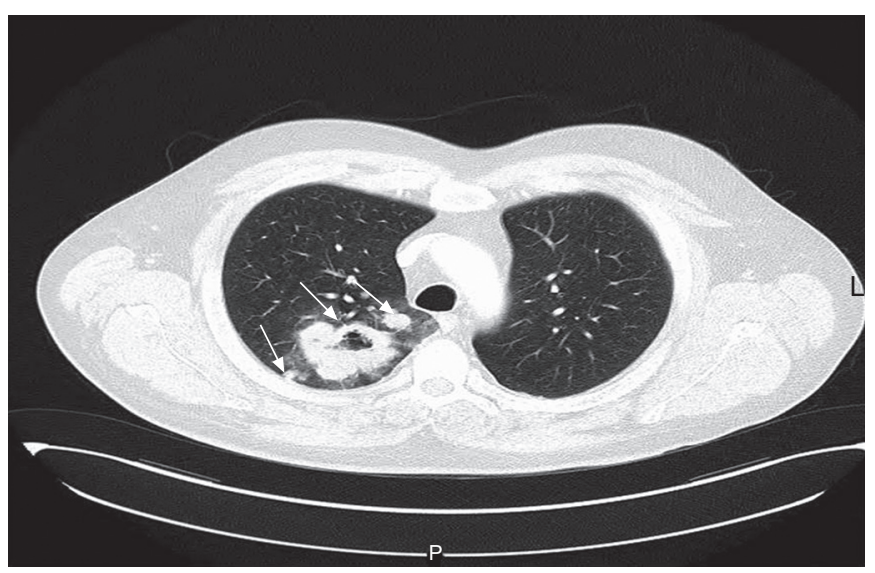

FIGURE 1. Computed tomography of the thorax showing right upper lobe thick-walled cavity with satellite lesions (arrows). showed an enlarging cavity that now encased the right main pulmonary artery. Given the significant haemoptysis, worsening radiological appearance and lack of definitive diagnosis, video-assisted thoracoscopic RUL resection was performed for diagnostic and therapeutic purposes. Multiple frozen-section specimens demonstrated no evidence of malignancy but showed prominent inflammation and vascular proliferation associated with fibrin deposition, suggesting a possible vasculitic process. However, final pathological examination of the resected lobe confirmed a high-grade neoplasm composed of sheets of large pleomorphic cells with numerous multinucleated giant cells and wreath-like cells, typical of an anaplastic large cell lymphoma (fig. 2). The adjacent lung parenchyma showed extensive cryptogenic organising pneumonia-like fibroblastic proliferation, prominent inflammatory infiltrate and secondary pulmonary vascular changes, corresponding to the frozensection sample findings. Immunohistochemistry showed CD4 and CD30 positivity, though anaplastic lymphoma kinase (ALK) staining was negative. An American Thoracic Society station 4R lymph node also showed tumour involvement. Although the patient received standard adjuvant chemotherapy with cyclophosphamide, doxorubicin, vincristine and prednisolone (CHOP), follow-up imaging confirmed widespread disease dissemination and he died 4 months after diagnosis.

Establishing a cause for pulmonary cavities through relatively noninvasive testing is often challenging, as highlighted by this case. The combination of haemoptysis, constitutional symptoms and an upper lobe cavity made primary lung malignancy, bacterial infection, tuberculosis and vasculitis the most likely diagnoses. Despite extensive investigation, however, a definitive cause could not be established prior to lobectomy. Diagnosis can be particularly challenging when malignant lesions become superinfected with pathogenic organisms [1].

Anaplastic lymphoma is a form of non-Hodgkin's lymphoma of T-lymphocyte or null cell origin, characterised by the presence of CD30 (Ki-1)-positive cells [2]. Overall, it accounts for 2-3\% of

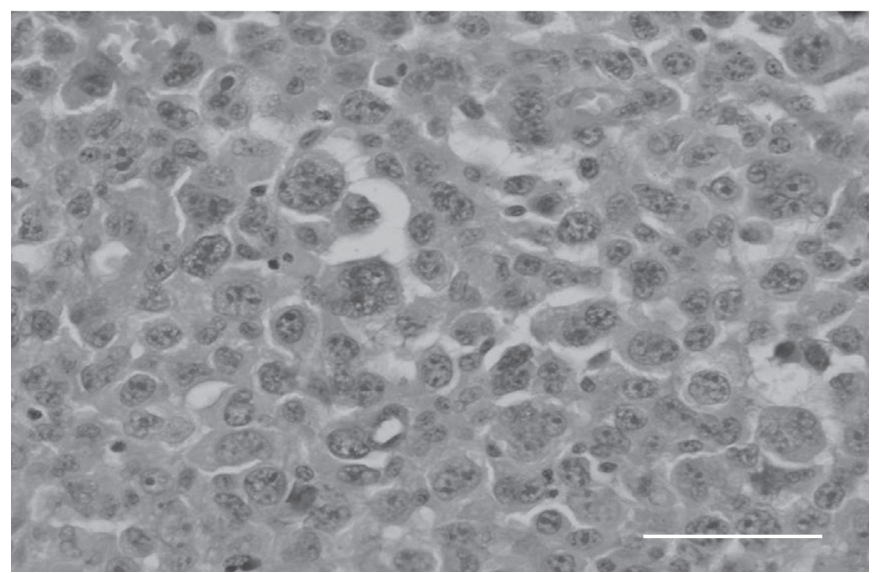

FIGURE 2. Anaplastic large cell lymphoma, with multinucleated tumour giant cells in the centre of image. Haematoxylin and eosin staining. Scale bar $=100 \mu \mathrm{m}$. 
lymphomas but affects the lung in only $5-15 \%$ of cases [3]. There is a male predominance and a bimodal age distribution, with peaks in children and the elderly. The disease is classified into cutaneous and systemic forms, the latter being more aggressive. Anaplastic lymphoma is further classified into ALK-positive and -negative subtypes. Although patients generally present with advanced (stage III or IV) disease, prognosis is usually favourable, particularly in ALK-positive tumours (overall survival 70-80\%) [4]. ALK-negative anaplastic lymphomas tend to occur in elderly patients and carry a worse prognosis [4].

Very few cases of primary pulmonary anaplastic lymphomas have previously been described. Cough, dyspnoea and fever are the most common presenting symptoms, with tracheal obstruction, mediastinal masses, lung masses, diffuse pulmonary infiltrates and a miliary nodular pattern being the usual radiological appearances [5]. Typical histological findings of anaplastic lymphomas are CD30-positive cells with abundant cytoplasm and prominent nucleoli [6]. Establishing a diagnosis is complicated by the wide morphological spectrum of neoplastic cells and, when sampling extranodal sites, by the florid adjacent reactive changes, as exemplified in this case. The initial lack of histological evidence of malignancy (even from open-lung biopsy specimens) in our patient was the result of sampling discrepancy, as only adjacent reactive changes were submitted for evaluation. Indeed, the finding of widespread vascular inflammatory changes initially raised the possibility of a pulmonary vasculitis, despite negative serological testing. Interestingly, an association between lymphomas and ANCA positivity has previously been reported, which adds further to the diagnostic confusion [7].

The treatment for systemic anaplastic lymphoma consists of combination cytotoxic chemotherapy (CHOP) with or without radiotherapy for bulky sites of disease. The observation that certain lung cancers express ALK has aroused interest in the development of therapies that specifically target ALK. Crizotinib, recently approved for lung adenocarcinoma, has also shown promise in some anaplastic lymphoma patients [8]. Other investigational treatments include autologous stem cell transplantation and anti-CD30 antibodies.

Pulmonary cavitation is a frequently encountered clinical problem. However, establishing a diagnosis through minimally invasive testing approaches that yield relatively small tissue samples for analysis can be difficult. Where diagnostic confusion remains, recourse to surgical biopsy should be considered, particularly where there is clinical worsening. Even so, sampling errors can occur, particularly when there is extensive surrounding inflammation, and a high index of clinical suspicion for uncommon causes of pulmonary malignancy should be maintained.

Caroline O'Connell*, Mardiana Abdul Aziz", John C. O'Keane", David Healy $^{\star}$ and Dermot S. O'Callaghan*

*Dept of Respiratory Medicine, Mater Misericordiae University Hospital, Dublin, "Dept of Pathology, Mater Misericordiae University Hospital, Dublin, and "Dept of Cardiothoracic Surgery, Mater Misericordiae University Hospital, Dublin, Ireland.

Correspondence: C. O'Connell, Dept of Respiratory Medicine, Mater Misericordiae University Hospital, Eccles Street, Dublin 7, Ireland. E-mail: caroline1597@hotmail.com

Statement of Interest: Conflict of interest information can be found alongside the online version of this article at err.ersjournals.com

Provenance: Submitted article, peer reviewed.

\section{REFERENCES}

1 Gadkowski LB, Stout JE. Cavitary pulmonary disease. Clin Microbiol Rev 2008; 21: 305-333.

2 Greer JP, Kinney MC, Collins RD, et al. Clinical features of 31 patients with Ki-1 anaplastic large-cell lymphoma. J Clin Oncol 1991; 9: 539-547.

3 Campo E, Chott A, Kinney MC, et al. Update on extranodal lymphomas. Conclusions of the Workshop held by the EAHP and the SH in Thessaloniki, Greece. Histopathology 2006; 48: 481-504.

4 Savage KJ, Harris NL, Vose JM, et al. ALK ${ }^{-}$anaplastic large-cell lymphoma is clinically and immunophenotypically different from both $\mathrm{ALK}^{+} \mathrm{ALCL}$ and peripheral T-cell lymphoma, not otherwise specified: report from the International Peripheral T-Cell Lymphoma Project. Blood 2008; 111: 5496-5504.

5 Rush WL, Andriko JA, Taubenberger JK, et al. Primary anaplastic large cell lymphoma of the lung: a clinicopathologic study of five patients. Mod Pathol 2000; 13: 1285-1292.

6 Chan JK, Kwong YL. Common misdiagnoses in lymphomas and avoidance strategies. Lancet Oncol 2010; 11: 579-588.

7 Cil T, Altintas A, Isikdogan A, et al. Prevalence of antineutrophil cytoplasmic antibody positivity in patients with Hodgkin's and nonHodgkin lymphoma: a single center experience. Int J Hematol 2009; 90: 52-57.

8 Gambacorti-Passerini C, Messa C, Pogliani E. Crizotinib in anaplastic large-cell lymphoma. N Engl J Med 2011; 364: 775-776.

DOI: $10.1183 / 09059180.00005012$ 